\title{
2nd Issue of Real-Time Image Processing
}

\author{
N. Kehtarnavaz $\cdot$ M. F. Carlsohn
}

Published online: 17 April 2009

(C) Springer-Verlag 2009

It is now over 3 years that Journal of Real-Time Image Processing (JRTIP) has been publishing papers solely dedicated to the real-time aspects of image and video processing. So far 14 issues in four volumes have successfully appeared. In volume 4, two special issues, one on "Real-Time Aspects of the H264 Family of Standards" already appeared as the first issue and the other one on "Architectures and Techniques for Real-Time Processing of Remotely Sensed Images" will appear as the third issue. It is encouraging to see that the number of downloads of papers from JRTIP was at the level of 15,200 by the end of 2008 indicating the growing importance of the real-time image processing area.

This second issue of the fourth volume comprises six original research articles, five of which address the importance of computer architectures for the real-time performance of image and video processing systems.

Noting the growing trend in using multi-core processors, the first paper presents an interesting 2D switch-based Network-on-Chip approach exploiting dynamic and partial reconfiguration in order to adapt system architecture to the actual requirements of image processing applications incorporating the processing elements as well as the communication infrastructure.

The second paper discusses a comparison of three architectures: a general purpose of RISC processor-the

N. Kehtarnavaz

University of Texas, Dallas, USA

M. F. Carlsohn ( $\square)$

Ingenieurberatung fuer Computer Vision

und Bildkommunikation, Am Heiddamm 36g,

28355 Bremen, Germany

e-mail: Matthias.Carlsohn@t-online.de
PowerPC G4 - a parallel artificial retina dedicated to lowlevel image processing-Pvlsar34-and the Associative Mesh, a specialized architecture based on associative net for the implementation of robust motion detection algorithms. The different aspects and constraints of embedded systems, execution time and power consumption of these architectures are compared from the perspective of "next generation" vision system requirements of embedded solutions.

The third paper presents a set of platform-independent architectural optimizations for improving the performance of software-based standard video coders demonstrated via an MPEG4 reference implementation targeting in particular execution time and memory usage identifying bottlenecks and adapting data flows accordingly, while leaving the coding algorithm unchanged and thus avoiding any impact on the coding results.

The fourth paper contributes to the field of FPGA architectures by a reconfigurable design that provides efficient interconnection structures for improvement of performance and power consumption, taking into consideration the statistical and spatial data extracted from applications such as multi-media and communications. An extensive comparison study with respect to several design parameters is presented to show the effectiveness of the proposed architecture.

The fifth paper deals with architectural issues by discussing the design and implementation of a video transcoding architecture. It supports a jerkiness driven variable frame rate control that is compatible with the constraints of real-time communication tested under a wide range of conditions. The motion of incoming video frames is traced and tuned to the outgoing frame rate according to the level of jerkiness acceptable by the user providing an optimum use of both the available bandwidth and terminal resources. 
Finally, the last paper is dedicated to a real fast image processing application involving a real-time motion analysis system for detecting the scratching behavior of laboratory mice. This system can detect in a non-invasive manner laboratory mice scratching by introducing a specially designed high-speed vision system that can calculate the frame-toframe difference at a frame rate of 240 frames per second.

We would like to use this opportunity to mention that the editorial board of JRTIP recently met in San Jose, CA, for the second time where they were attending the SPIE Conference on Real-Time Image and Video Processing. A picture of some of the editorial board members and guest editors present at the Springer sponsored dinner meeting is shown below. The following three special issues were formulated at this meeting: "Real-Time Biometrics and Secure Media", "Real-Time Vision-Based Motion Analysis and Intelligent Transportation Systems" and "Parallel Computing for Real-Time Image Processing". The corresponding calls for papers can be found at the end of this issue. In addition, a call for papers regarding the SPIE Conference on Real-time Image and Video Processing is also listed which is to take place for the first time in Brussels (Belgium) in conjunction with the SPIE's Photonics Europe Symposium in April 2010. It is planned to alternate this conference between Europe and the USA to enable participation at a higher level by European researchers.

We would like to welcome the following three new members to the editorial board who have already made

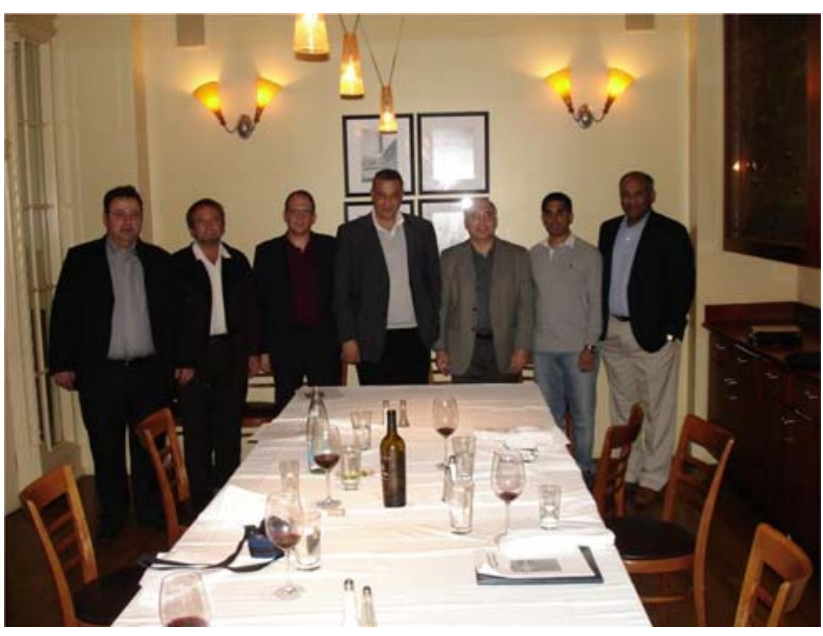

Fig. 1 Christos Grecos, Volodymyr Ponomaryov, Barak Fishbain, Mohammad Akil, Nasser Kehtarnavaz, Mark Gamadia and Mukul Shirvaikar (from left to right)

contributions to JRTIP by serving as guest editors and reviewers: Dr. Christos Grecos, Dr. Sergio Goma and Dr. Antonio Plaza.

At the end, we wish to express our gratitude to the Associate Editors and the reviewers for their continued contributions. We also appreciate the help and support that the Editorial Office of Springer has been providing JRTIP (Fig. 1). 